\title{
Magnetic phase diagram of Ca-substituted $\mathrm{EuFe}_{2} \mathrm{As}_{2}$
}

\author{
L. M. Tran,,${ }^{1, *}$ M. Babij, ${ }^{1}$ L. Korosec, ${ }^{2}$ T. Shang, ${ }^{3,4}$ Z. Bukowski, ${ }^{1}$ and T. Shiroka ${ }^{2,3}$ \\ ${ }^{1}$ Institute of Low Temperature and Structure Research, Polish Academy of Sciences, P.O. Box 1410, PL-50-422 Wroclaw, Poland \\ ${ }^{2}$ Laboratorium für Festkörperphysik, ETH Zürich, CH-8093 Zurich, Switzerland \\ ${ }^{3}$ Paul Scherrer Institut, CH-5232 Villigen PSI, Switzerland \\ ${ }^{4}$ Institute of Condensed Matter Physics, École Polytechnique Fédérale de Lausanne (EPFL), Lausanne CH-1015, Switzerland
}

(Received 6 April 2018; revised manuscript received 13 July 2018; published 11 September 2018)

\begin{abstract}
The simultaneous presence of an Fe-related spin-density wave and the antiferromagnetic order of $\mathrm{Eu}^{2+}$ moments ranks $\mathrm{EuFe}_{2} \mathrm{As}_{2}$ among the most interesting parent compounds of iron-based pnictide superconductors. Here we explore the consequences of the dilution of $\mathrm{Eu}^{2+}$ magnetic lattice through on-site Ca substitution. By employing macro- and microscopic techniques, including electrical transport and magnetometry, as well as muon-spin spectroscopy, we study the evolution of Eu magnetic order in both the weak and strong dilution regimes, achieved for Ca concentration $x(\mathrm{Ca})=0.12$ and 0.43 , respectively. We demonstrate the localized character of the Eu antiferromagnetism mediated via RKKY interactions, in contrast with the largely itinerant nature of Fe magnetic interactions. Our results suggest a weak coupling between the Fe and Eu magnetic sublattices and a rapid decrease of the Eu magnetic interaction strength upon Ca substitution. The latter is confirmed both by the depression of the ordering temperature of the $\mathrm{Eu}^{2+}$ moments $T_{\mathrm{N}}$, and the decrease of magnetic volume fraction with increasing $x(\mathrm{Ca})$. We establish that, similarly to the $\mathrm{EuFe}_{2} \mathrm{As}_{2}$ parent compound, the investigated Ca-doped compounds have a twinned structure and undergo a permanent detwinning upon applying an external magnetic field.
\end{abstract}

DOI: 10.1103/PhysRevB.98.104412

\section{INTRODUCTION}

Superconductivity and magnetic order are among the most studied topics in contemporary solid-state physics. Although commonly considered to be antagonistic phenomena, in certain cases superconductivity has been observed to coexist with magnetic order [1]. In this respect, iron-based superconductors (Fe-SC) are among the most recent examples to exhibit such an interplay between superconductivity and magnetism [2,3]. In particular, $\mathrm{EuFe}_{2} \mathrm{As}_{2}$-based compounds possess some unique properties, reflecting the simultaneous occurrence of superconductivity and of two separate magnetically ordered subsystems (due to iron and europium layers) [4,5]. In the $\mathrm{EuFe}_{2} \mathrm{As}_{2}$ parent compound, a spin-density-wave (SDW) order associated with the Fe $3 d$ electrons is observed at $T_{\mathrm{SDW}} \approx 190 \mathrm{~K}$. The SDW transition is accompanied by a structural phase transition from the tetragonal $(I 4 / \mathrm{mmm})$ to the orthorhombic $(F m m m)$ structure. Additionally, europium magnetic moments order antiferromagnetically at $T_{\mathrm{N}}=19 \mathrm{~K}$ in an A-type magnetic structure. This corresponds to magnetic moments being aligned ferromagnetically along the $a$ axis, and antiferromagnetically along the $c$ axis [6]. Superconductivity in the $\mathrm{EuFe}_{2} \mathrm{As}_{2}$-based compounds emerges when the magnetic order of the itinerant $\mathrm{Fe} 3 d$ electrons gets suppressed, either via applied pressure [7-9] or chemical substitution [4]. The latter involves either isovalent substitutions in the FeAs-layers [5,10-14] or electron/hole doping in the Eu layers [15-17].

*Corresponding author: 1.m.tran@int.pan.wroc.pl
Upon substitution in the FeAs layers, the SDW ordering temperature decreases compared to the $T_{\mathrm{SDW}}$ of the parent compound, with SDW order being completely suppressed in the overdoped compounds. For Co-doped compounds it was reported that chemical substitution modifies the shape of the SDW [18], yet the doping does not change the magnetic ground state of the $\mathrm{Fe}^{2+}$ magnetic moments, which remains antiferromagnetic as long as the order is present [19]. The magnetic ground state of the Eu subsystem, however, changes from an A-type antiferromagnet, through a canted antiferromagnet, to a ferromagnet upon doping the FeAs sublattice [19-21].

A rather different behavior is observed for isovalent substitution of europium. On one hand, the $\mathrm{Ca}$ doping does not lead to superconductivity under ambient pressure since the $\mathrm{Fe}$ related SDW remains nearly unchanged, i.e., the substitution does not modify significantly either the shape of SDW, or its ordering temperature [22-25]. On the other hand, the dilution of the Eu sublattice with nonmagnetic $\mathrm{Ca}^{2+}$ ions decreases the temperature of the antiferromagnetic order, eventually leading to its disappearance for Eu dilutions above 50\% [23,24]. Thus, we expect the interactions between $\mathrm{Eu}^{2+}$ ions to change significantly upon $\mathrm{Ca}$ doping, with $\mathrm{Eu}_{1-x} \mathrm{Ca}_{x} \mathrm{Fe}_{2} \mathrm{As}_{2}$ representing an ideal system for studying the Eu-related magnetism in the $\mathrm{EuFe}_{2} \mathrm{As}_{2}$-based compounds and its possible interplay with $\mathrm{Fe}$ magnetism, while keeping the SDW order unchanged.

To shed light on the magnetic phase transitions of such a system, we investigated the zero-field resistivity, ac susceptibility, dc magnetization, and muon-spin relaxation $(\mu \mathrm{SR})$ of two Ca-doped $\mathrm{EuFe}_{2} \mathrm{As}_{2}$ compounds. It is worth noting that the $\mu \mathrm{SR}$ spectroscopy has been successfully used to investigate other $\mathrm{EuFe}_{2} \mathrm{As}_{2}$-based compounds, for which it could reveal the nature of magnetic order, the magnetic phase diagram, and 
the internal field values $[20,26,27]$. Unlike previous studies, where only the effects of $\mathrm{Fe}$ magnetic lattice dilution or As isoelectronic replacement were considered, here we investigate the microscopic consequences of the Eu magnetic lattice dilution via on-site substitution with nonmagnetic $\mathrm{Ca}^{2+}$ ions. To date, no microscopic studies of the Ca-doped $\mathrm{EuFe}_{2} \mathrm{As}_{2}$ system exist. By using the $\mu$ SR technique we aimed to explore the interplay of Fe-3d and Eu- $4 f$ magnetism, as well as a possible coupling between the two, with a particular focus on the low-temperature antiferromagnetic region, dominated by the rare-earth magnetic interactions.

\section{EXPERIMENTAL DETAILS}

Single crystals of $\mathrm{Eu}_{1-x} \mathrm{Ca}_{x} \mathrm{Fe}_{2} \mathrm{As}_{2}$ were grown using the Sn-flux method. The Eu, Fe, As, Ca, and Sn elements in molar ratios of $(1-x): 2: 2: x: 30$ were loaded into alumina crucibles and sealed in quartz ampules under vacuum. To dissolve all the ingredients, the ampules were heated slowly to $1050{ }^{\circ} \mathrm{C}$, kept at this temperature for several hours, and then cooled down slowly to $650^{\circ} \mathrm{C}$ with a rate of $2{ }^{\circ} \mathrm{C} / \mathrm{h}$. Next, the liquid tin was decanted from the crucibles. The $\mathrm{Sn}$ residues on the crystals were removed via etching in diluted hydrochloric acid.

The chemical composition of the obtained crystals was determined using the energy-dispersive x-ray spectroscopy (EDX), whereas the crystal structure and phase purity were characterized by powder $\mathrm{x}$-ray diffraction (XRD) using an $X$ 'Pert Pro powder diffractometer (PANalytical, The Netherlands) equipped with a linear PIXcel detector.

The ac magnetic susceptibility measurements were performed using an Oxford Instruments susceptometer in the temperature range $2-300 \mathrm{~K}$ and external fields up to $2 \mathrm{~T}$, probing with a driving field $\mu_{0} H_{\mathrm{ac}} \simeq 1 \mathrm{mT}$ at a frequency $f=1.111 \mathrm{kHz}$. The dc magnetization measurements were carried out in a Physical Properties Measurement System (PPMS, Quantum Design) in magnetic fields up to $9 \mathrm{~T}$ and covering a temperature range of $2-320 \mathrm{~K}$. The magnetization data were collected in both zero-field-cooling (ZFC) and fieldcooling (FC) modes, whereas the ac magnetic susceptibility measurements were performed in ZFC mode only. Both dc and ac measurements were conducted with the external fields applied parallel as well as perpendicular to the $c$ axis.

Resistivity data were collected using the PPMS platform in the temperature range from 2 to $300 \mathrm{~K}$. Silver-wire contacts were mounted on the surface parallel to the crystallographic $a b$ plane using DuPont conductive silver paint; the contact resistances were less than $0.5 \Omega$.

The $\mu$ SR measurements were carried out at the General Purpose Spectrometer (GPS) at the $\pi \mathrm{M} 3$ beamline of the Swiss Muon Source at the Paul Scherrer Institut (PSI) in Villigen, Switzerland. The temperature dependence of the muon-spin relaxation was investigated both in zero-(ZF) and in longitudinally (LF) applied magnetic fields. Several single crystals, 0.5 - to $1-\mathrm{mm}$ thick were arranged in a mosaic configuration with their $c$ axes collinear on an aluminated mylar sheet. The latter was then folded to form an envelope on top of which suitable 50- $\mu \mathrm{m}$ thick kapton foils were added to optimize the muon stopping rate. The sample was finally mounted on a copper fork (fly-past setup) with a negligible

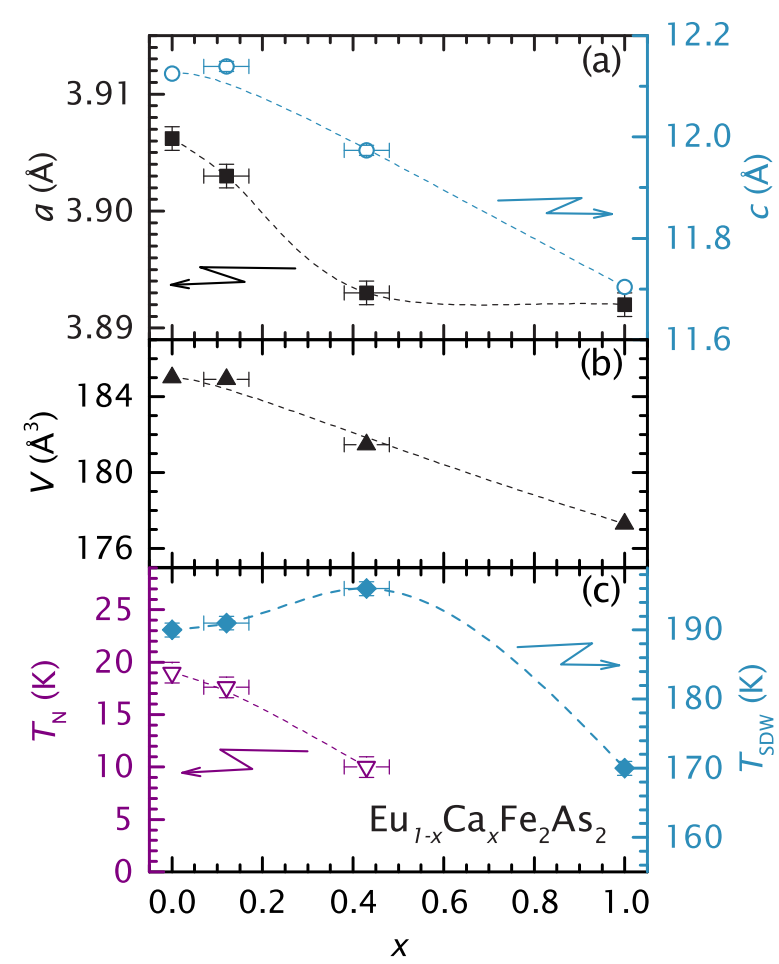

FIG. 1. Dependence of (a) $a$ and $c$ lattice parameters, (b) unit cell volume $V$, and (c) $T_{\mathrm{N}}$ and $T_{\mathrm{SDW}}$ temperatures on the Ca-concentration $x$ in $\mathrm{Eu}_{1-x} \mathrm{Ca}_{x} \mathrm{Fe}_{2} \mathrm{As}_{2}$. Dashed lines are guides to the eye.

background count. Active-field compensation during ZF- $\mu$ SR measurements ensured a stray field value below $1 \mu \mathrm{T}$. Data in both ZF and LF experiments were collected in a temperature range of 1.6 to $60 \mathrm{~K}$ and, occasionally, up to $240 \mathrm{~K}$ (to confirm the known Fe spin-density wave at approximately $190 \mathrm{~K}$ ). For the LF experiments magnetic fields up to $0.6 \mathrm{~T}$ were applied.

\section{EXPERIMENTAL RESULTS AND DISCUSSION}

\section{A. Composition and crystal structure}

For the current study we prepared single crystals of $\mathrm{EuFe}_{2} \mathrm{As}_{2}$ and $\mathrm{CaFe}_{2} \mathrm{As}_{2}$, as well as of $\mathrm{Eu}_{0.88} \mathrm{Ca}_{0.12} \mathrm{Fe}_{2} \mathrm{As}_{2}$ and $\mathrm{Eu}_{0.57} \mathrm{Ca}_{0.43} \mathrm{Fe}_{2} \mathrm{As}_{2}$. All the observed $\mathrm{x}$-ray diffraction reflections for the investigated samples could be indexed to the tetragonal $\mathrm{ThCr}_{2} \mathrm{Si}_{2}$-type structure (I4/ $\mathrm{mmm}$ space group), expected for the $A \mathrm{Fe}_{2} \mathrm{As}_{2}$-based systems. The refined $a$ and $c$ lattice parameters and the calculated unit cell volumes $V$ are shown in Figs. 1(a) and 1(b). Both lattice parameters (and the unit cell volume) decrease with increasing $\mathrm{Ca}$ concentration and are in good agreement with those reported by Mitsuda et al. [24].

\section{B. Electrical resistivity}

The temperature dependencies of resistivity normalized to the 300-K resistivity values for the whole $\mathrm{Eu}_{1-x} \mathrm{Ca}_{x} \mathrm{Fe}_{2} \mathrm{As}_{2}$ series $(x=0,0.12,0.43$, and 1) are shown in Fig. 2. For all the compositions a pronounced anomaly is observed at the spin-density-wave (SDW) ordering temperature of the $\mathrm{Fe}$ conduction electrons $\left(T_{\mathrm{SDW}}\right)$, coinciding with a tetragonal-toorthorhombic structural phase transition. The $T_{\mathrm{SDW}} \sim 190 \mathrm{~K}$ 


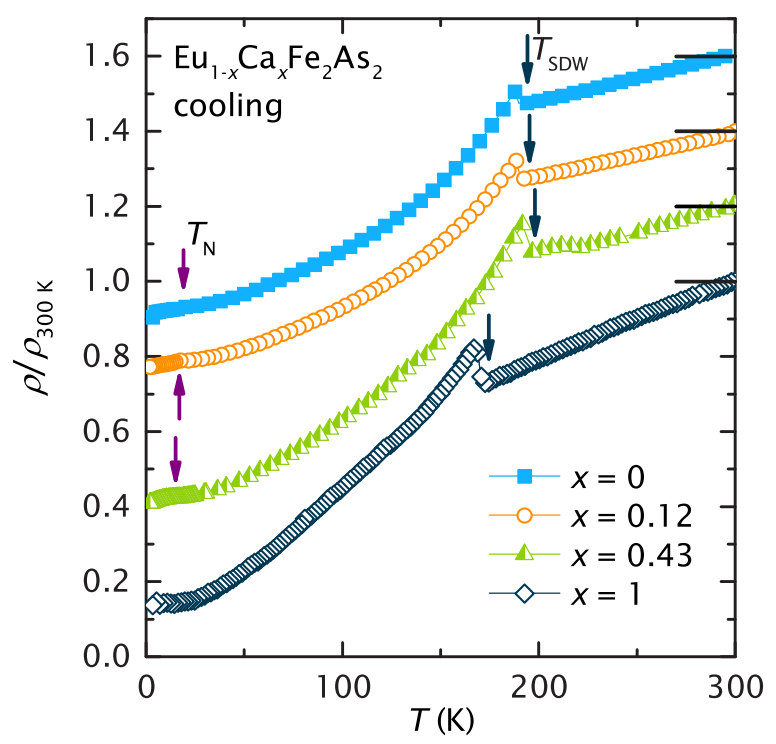

FIG. 2. Normalized resistivity $\rho / \rho_{300 \mathrm{~K}}$ vs temperature $T$ for the $\mathrm{Eu}_{1-x} \mathrm{Ca}_{x} \mathrm{Fe}_{2} \mathrm{As}_{2}$ series with $x=0,0.12,0.43$, and 1 . The $T_{\mathrm{N}}$ and $T_{\mathrm{SDw}}$ temperatures are marked with arrows. For clarity, the resistivity curves for $x>0$ are shifted vertically by 0.2 units, with the horizontal lines marking the respective $\rho / \rho_{300 \mathrm{~K}}=1$ levels.

is nearly the same for all compounds, except for $\mathrm{CaFe}_{2} \mathrm{As}_{2}$ $\left(T_{\mathrm{SDW}} \sim 170 \mathrm{~K}\right)$, in good agreement with the literature data $[23,24,28]$.

A much weaker anomaly, corresponding to the antiferromagnetic ordering of $\mathrm{Eu}^{2+}$ magnetic moments, is observed below $19 \mathrm{~K}$. As expected, the dilution of the Eu magnetic sublattice (via nonmagnetic $\mathrm{Ca}$ ions) leads to a decreased Néel temperature $T_{\mathrm{N}}$. The observed $T_{\mathrm{N}}$ for $\mathrm{Eu}_{1-x} \mathrm{Ca}_{x} \mathrm{Fe}_{2} \mathrm{As}_{2}$ are in good agreement with those reported by Mitsuda et al. [24]. The evolution of both the $T_{\mathrm{SDW}}$ and $T_{\mathrm{N}}$ values with Ca concentration is summarized in Fig. 1(c).

\section{Magnetization}

The field-dependent magnetization $M(H)$ of both $12 \%$ and 43\% Ca-doped compounds was investigated in external magnetic fields applied either parallel (not shown) or perpendicular (Figs. 3 and 4) to the $c$ axis. The measurements were carried out in both increasing and decreasing fields. No spontaneous magnetization was detected in any of the compounds and the initial slope is linear, as expected for antiferromagnetic systems. Magnetic hysteresis was observed for both systems, but only for fields applied perpendicular to the $c$ axis and at temperatures below $5 \mathrm{~K}$.

The $M(H)$ curves measured in the magnetic fields applied perpendicular to the $c$ axis are presented in Fig. 3. At $T=2 \mathrm{~K}$ the magnetization of both $\mathrm{Eu}_{0.88} \mathrm{Ca}_{0.12} \mathrm{Fe}_{2} \mathrm{As}_{2}$ and $\mathrm{Eu}_{0.57} \mathrm{Ca}_{0.43} \mathrm{Fe}_{2} \mathrm{As}_{2}$ is characterized by a linear initial slope, a small magnetic hysteresis at $0.2 \mathrm{~T}$, and a metamagnetic transition at 0.6 and $0.7 \mathrm{~T}$, respectively (Fig. 4). In the $\mathrm{Eu}_{0.88} \mathrm{Ca}_{0.12} \mathrm{Fe}_{2} \mathrm{As}_{2}$ case, an additional hysteresis is observed at $\sim 0.6 \mathrm{~T}$ [see Fig. 4(a)].

As can be seen in Fig. 4(a), the field-dependent magnetization of $\mathrm{Eu}_{0.88} \mathrm{Ca}_{0.12} \mathrm{Fe}_{2} \mathrm{As}_{2}$ changes from linear (first field increase) to one with a positive curvature (subsequent measure-
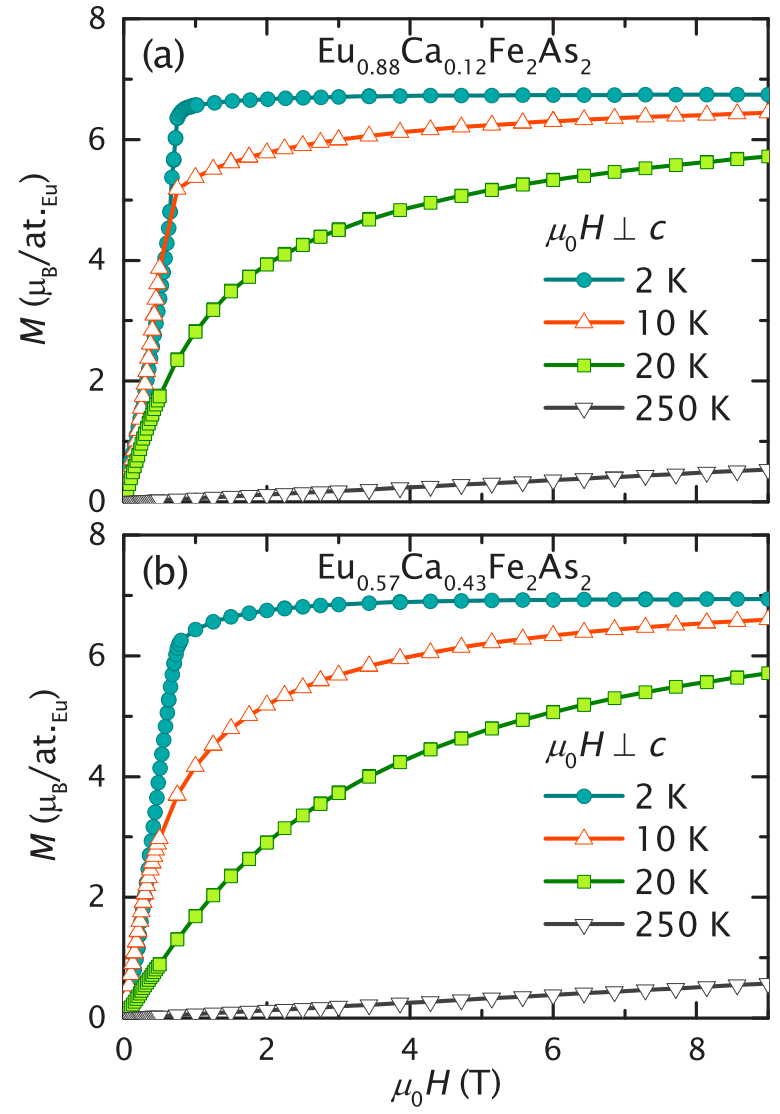

FIG. 3. Magnetic field dependence of magnetization per Eu atom for (a) $\mathrm{Eu}_{0.88} \mathrm{Ca}_{0.12} \mathrm{Fe}_{2} \mathrm{As}_{2}$ and (b) $\mathrm{Eu}_{0.57} \mathrm{Ca}_{0.43} \mathrm{Fe}_{2} \mathrm{As}_{2}$ measured at selected temperatures and in increasing fields applied perpendicular to the $c$ axis.

ments), suggesting the presence of ferromagnetic correlations. On the other hand, a linear dependence up to $0.1 \mathrm{~T}$ is observed in $\mathrm{Eu}_{0.57} \mathrm{Ca}_{0.43} \mathrm{Fe}_{2} \mathrm{As}_{2}$ for both increase- and decrease-field measurements [Fig. 4(b)].

For both investigated compounds the low-field hysteresis (at $\sim 0.2 \mathrm{~T}$ ) disappears in subsequently repeated increase- and decrease-field measurements and the $M(H)$ curves follow the same dependence as the first decrease-fields dependence (cf. solid lines in Fig. 4). Additionally, in the $\mathrm{Eu}_{0.57} \mathrm{Ca}_{0.43} \mathrm{Fe}_{2} \mathrm{As}_{2}$ case, the width of the high-field hysteresis loop (at $\sim 0.6 \mathrm{~T}$ ) decreases in the subsequent measurements. Moreover, the initial field dependence of magnetization can only be reproduced after heating the samples above the respective $T_{\mathrm{SDW}}$.

A similar field dependent magnetization was found in the $\mathrm{EuFe}_{2} \mathrm{As}_{2}$ parent compound. As shown by Jiang et al. [29], when an external magnetic field is applied perpendicular to the $c$ axis, subsequent metamagnetic transitions are observed. Neutron-spectroscopy studies have revealed that $\mathrm{EuFe}_{2} \mathrm{As}_{2}$ crystals have a twinned structure, whereby the application of an external magnetic field modifies the twinning fraction, at fields corresponding to the metamagnetic transitions [6]. A study by Zapf et al. [30] suggests that both the metamagnetic transitions and the hysteretic behavior are due to "detwinning." There it was shown not only that $\mathrm{EuFe}_{2} \mathrm{As}_{2}$ undergoes two detwinning processes (at 0.1 and at $1 \mathrm{~T}$ ), but also that the detwinning 

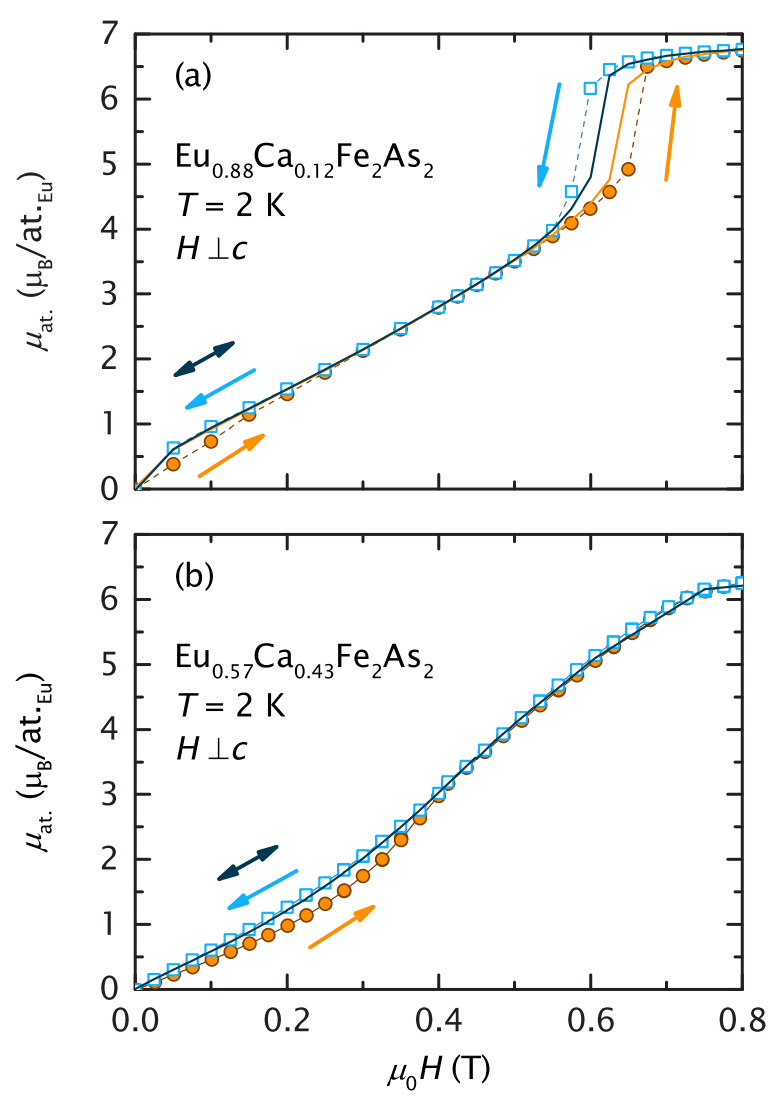

FIG. 4. Field dependence of (a) $\mathrm{Eu}_{0.88} \mathrm{Ca}_{0.12} \mathrm{Fe}_{2} \mathrm{As}_{2}$ and (b) $\mathrm{Eu}_{0.57} \mathrm{Ca}_{0.43} \mathrm{Fe}_{2} \mathrm{As}_{2}$ magnetization (in units of magnetic moment per Eu atom) measured at $2 \mathrm{~K}$ in increasing (full circles) and decreasing (open squares) external magnetic fields applied perpendicular to the $c$ axis. Thick solid lines represent the magnetization measured in the second run (see text) in increasing/decreasing fields.

is permanent as long as the sample is not heated above $T_{\text {SDW }}$. A recent theoretical study indicates that the detwinning in magnetic fields is characteristic of Fe-SC systems [31]. However, in $\mathrm{EuFe}_{2} \mathrm{As}_{2}$-based systems, the fields allowing for a persistent detwinning are much smaller than those required in other $A \mathrm{Fe}_{2} \mathrm{As}_{2}$ systems $(A=\mathrm{Ba}, \mathrm{Ca}, \ldots)$, thus reflecting the large-spin Eu ions and the coupling of the $\mathrm{Fe}$ and Eu sublattices.

The close resemblance of the $\mathrm{Eu}_{0.88} \mathrm{Ca}_{0.12} \mathrm{Fe}_{2} \mathrm{As}_{2}$ magnetization curve to that of the undoped $\mathrm{EuFe}_{2} \mathrm{As}_{2}$ suggests that the $12 \% \mathrm{Ca}$-doped compound undergoes a similar detwinning transition as the $\mathrm{EuFe}_{2} \mathrm{As}_{2}$ parent compound. By contrast, the magnetization of the $\mathrm{Eu}_{0.57} \mathrm{Ca}_{0.43} \mathrm{Fe}_{2} \mathrm{As}_{2}$ compound exhibits only the low-temperature magnetic hysteresis. Additionally, its $M(H)$ dependence exhibits a negative curvature in the rebound field, contrary to the $\mathrm{Eu}_{0.88} \mathrm{Ca}_{0.12} \mathrm{Fe}_{2} \mathrm{As}_{2}$ positive curvature. Remarkably, such intermediate metamagnetic states were not observed in the magnetization curves of systems with substituted FeAs sublattice that possess the canted-AF structure of $\mathrm{Eu}^{2+}$ magnetic moments [5,10,18].

For each magnetization isotherm measured at $T<T_{\mathrm{N}}$, the saturation magnetization can be determined. For instance, at $2 \mathrm{~K}$ the saturation magnetization $\mu_{\text {sat }}$ corresponds to $7 \mu_{\mathrm{B}}$, which is the expected theoretical value for magnetic $\mathrm{Eu}^{2+}$ ions ( $\mu_{\text {sat }}^{\text {th }}=g J$, with $g=2$ and $J=7 / 2$ ). It was shown that in

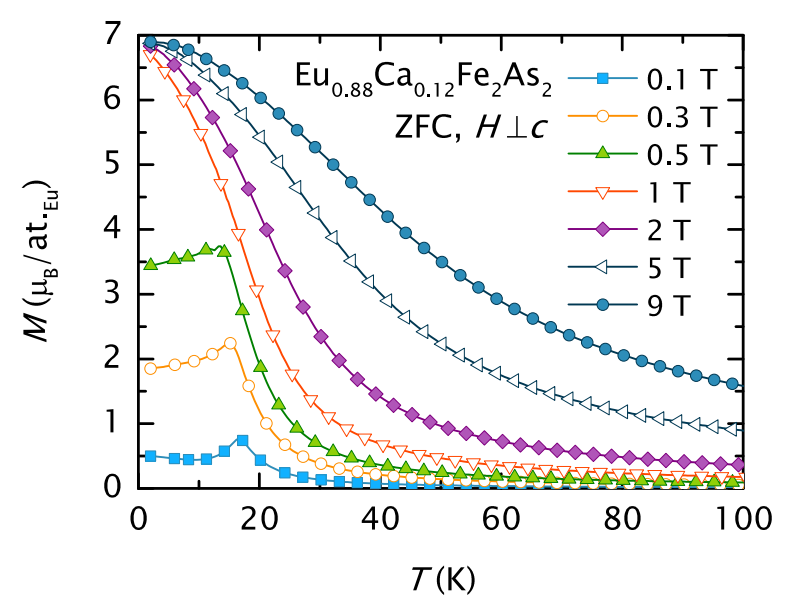

FIG. 5. Temperature dependence of $\mathrm{Eu}_{0.88} \mathrm{Ca}_{0.12} \mathrm{Fe}_{2} \mathrm{As}_{2}$ magnetization measured in ZFC mode in several magnetic fields $\left(\mu_{0} H\right)$ applied perpendicular to the $c$ axis.

$\mathrm{EuFe}_{2} \mathrm{As}_{2}$-based systems the saturation state is equivalent to a field-induced ferromagnetic state [5,11,29,32]. The field at which the system "switches" from an antiferromagnetic (AF) to a field-induced ferromagnetic state (FI-FM) is represented by the "crossover" field $H_{\mathrm{cr}}$. Such $H_{\mathrm{cr}}$ values can, therefore, be calculated by determining the minimum in the second derivative of magnetization $d^{2} M / d H^{2}$.

Figure 5 shows the temperature dependence of $\mathrm{Eu}_{0.88} \mathrm{Ca}_{0.12} \mathrm{Fe}_{2} \mathrm{As}_{2}$ ZFC magnetization measured at some representative fields applied perpendicular to the $c$ axis. As expected for an AF transition, the maximum in $M(T)$, corresponding to the ordering temperature of $\mathrm{Eu}^{2+}$ magnetic moments (e.g., at $T_{\mathrm{N}}=15.6 \mathrm{~K}$ in $0.1 \mathrm{~T}$ ), shifts towards lower temperatures with increasing magnetic field. Hence $T_{\mathrm{N}}$ was determined by using the Fisher's method-i.e., from the maximum of the derivative of the $T \chi(T)$ product [33]. Note, however, that for fields above $0.5 \mathrm{~T}, M(T)$ does not show a maximum, but only a smooth increase with decreasing temperature (up to $M \sim 7 \mu_{\mathrm{B}} / \mathrm{Eu}$ close to $T=0 \mathrm{~K}$ ).

In contrast to the Eu-related $\mathrm{AF}$ transition, the anomaly associated with the Fe-SDW transition is barely visible in the magnetization measurements. And when so, it can be observed only at high magnetic fields $\left(\mu_{0} H>2 \mathrm{~T}\right)$, as marked with blue vertical arrows in Fig. 6. These results are different from those of resistivity, where the SDW transition is much more prominent than the AF transition.

The 9-T dc magnetization data were used to calculate the magnetic susceptibility $\chi=M / H$. A typical temperature dependence of susceptibility and of its inverse for $\mathrm{Eu}_{0.88} \mathrm{Ca}_{0.12} \mathrm{Fe}_{2} \mathrm{As}_{2}$ in $H \| c$ are shown in Fig. 6. In the $100-180 \mathrm{~K}$ temperature range, the $\chi(T)$ susceptibility curves of $\mathrm{Eu}_{0.88} \mathrm{Ca}_{0.12} \mathrm{Fe}_{2} \mathrm{As}_{2}$ and $\mathrm{Eu}_{0.57} \mathrm{Ca}_{0.43} \mathrm{Fe}_{2} \mathrm{As}_{2}$, measured in magnetic fields parallel and perpendicular to the $c$ axis, could be fitted by means of a modified Curie-Weiss law:

$$
\chi=\frac{N_{\mathrm{A}}}{3 k_{\mathrm{B}}} \frac{\mu_{\mathrm{eff}}^{2}}{T-\theta_{\mathrm{p}}}+\chi_{0} .
$$

Here $N_{\mathrm{A}}$ is the Avogadro's constant, $k_{\mathrm{B}}$ is the Boltzmann constant, $\theta_{\mathrm{p}}$ is the Weiss temperature, and $\chi_{0}$ is a temperatureindependent paramagnetic susceptibility. The fit parameters in 


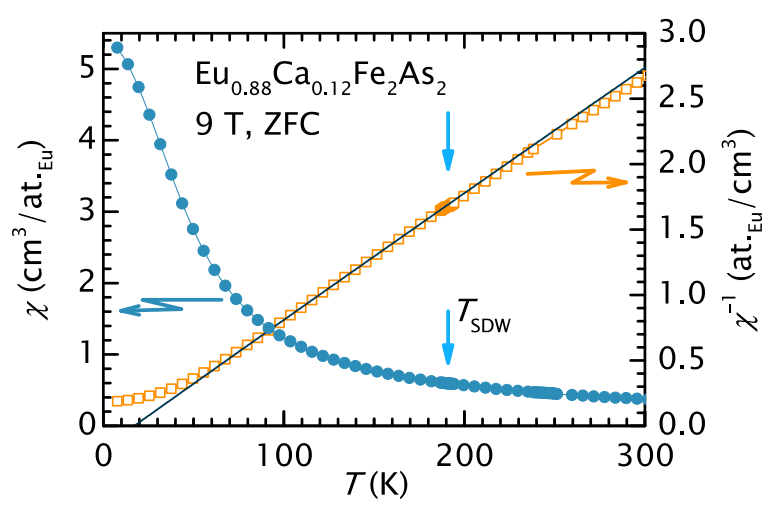

FIG. 6. Temperature-dependent ZFC magnetic susceptibility $\chi(T)$ (full circles, left axis) and its inverse $1 / \chi$ (open squares, right axis) for $\mathrm{Eu}_{0.88} \mathrm{Ca}_{0.12} \mathrm{Fe}_{2} \mathrm{As}_{2}$ measured in a 9-T magnetic field applied parallel to the $c$ axis. The solid line represent the fit by means of a modified Curie-Weiss law [Eq. (1)] in the 100-180 K temperature range. The $T_{\mathrm{SDW}}$ is marked with an arrow.

both cases are reported in Table I. The evaluated effective magnetic moments per Eu atom $\mu_{\text {eff }}$ have slightly larger values for measurements in magnetic fields applied parallel to the $c$ axis than for measurements with $H \perp c$. This was observed also in other $\mathrm{EuFe}_{2} \mathrm{As}_{2}$-based systems [4,5,10,11,29]. The determined $\mu_{\text {eff }} \sim 8 \mu_{\mathrm{B}}$ is marginally larger than $\mu_{\text {eff }}^{\text {theo }}=g \sqrt{J(J+1)}=$ $7.9 \mu_{\mathrm{B}}$ expected from theory, which is also consistent with previously reported results [4] and is usually attributed to a possible contribution of $\mathrm{Fe}^{2+}$ magnetic moments. The constant $\chi_{0}$ contribution is negligibly small when compared to the main magnetic susceptibility, confirming the absence of spurious phases.

Positive Weiss temperatures $\theta_{\mathrm{p}}$ suggest dominant ferromagnetic interactions in both compounds. Similar results were obtained in $\mathrm{EuFe}_{2} \mathrm{As}_{2}$ [29] and $\mathrm{EuCo}_{2} \mathrm{As}_{2}$ [34] too, most likely indicating also there the presence of ferromagnetic interactions between nearest-neighbor $\mathrm{Eu}^{2+}$ ions in the $a b$ planes.

\section{D. ac susceptibility}

The temperature dependence of the real part of ac susceptibility $\chi^{\prime}$ of $\mathrm{Eu}_{0.88} \mathrm{Ca}_{0.12} \mathrm{Fe}_{2} \mathrm{As}_{2}$ and $\mathrm{Eu}_{0.57} \mathrm{Ca}_{0.43} \mathrm{Fe}_{2} \mathrm{As}_{2}$ measured at selected $H \perp c$ fields is shown in Fig. 7. The zero-field data show a rather wide peak associated with the $\mathrm{AF}$ ordering of $\mathrm{Eu}^{2+}$ ions (at $T_{\mathrm{N}} \approx 15.6 \mathrm{~K}$ for $\mathrm{Eu}_{0.88} \mathrm{Ca}_{0.12} \mathrm{Fe}_{2} \mathrm{As}_{2}$ and at $10 \mathrm{~K}$ for $\mathrm{Eu}_{0.57} \mathrm{Ca}_{0.43} \mathrm{Fe}_{2} \mathrm{As}_{2}$ ). As expected for an $\mathrm{AF}$ transition, the peak shifts towards lower temperatures as the external magnetic field increases.

TABLE I. Magnetic parameters of $\mathrm{Eu}_{0.88} \mathrm{Ca}_{0.12} \mathrm{Fe}_{2} \mathrm{As}_{2}$ and $\mathrm{Eu}_{0.57} \mathrm{Ca}_{0.43} \mathrm{Fe}_{2} \mathrm{As}_{2}$, as evaluated from fitting the temperaturedependent susceptibility in $H \perp c$ and $H \| c$, using Eq. (1).

\begin{tabular}{lcccc}
\hline \hline \multirow{2}{*}{ Compound } & \multicolumn{2}{c}{$\mathrm{Eu}_{0.88} \mathrm{Ca}_{0.12} \mathrm{Fe}_{2} \mathrm{As}_{2}$} & \multicolumn{2}{c}{$\mathrm{Eu}_{0.57} \mathrm{Ca}_{0.43} \mathrm{Fe}_{2} \mathrm{As}_{2}$} \\
Orientation & $H \perp c$ & $H \| c$ & $H \perp c$ & $H \| c$ \\
\hline$\mu_{\text {eff }}\left(\mu_{\mathrm{B}}\right)$ & 8.1 & 8.2 & 7.9 & 8.2 \\
$\theta_{\mathrm{p}}(\mathrm{K})$ & 17.1 & 13.9 & 11.9 & 7.8 \\
$\chi_{0}\left(\mathrm{~cm}^{3} / \mathrm{Eu}\right)$ & 0.005 & - & 0.025 & 0.008 \\
\hline \hline
\end{tabular}
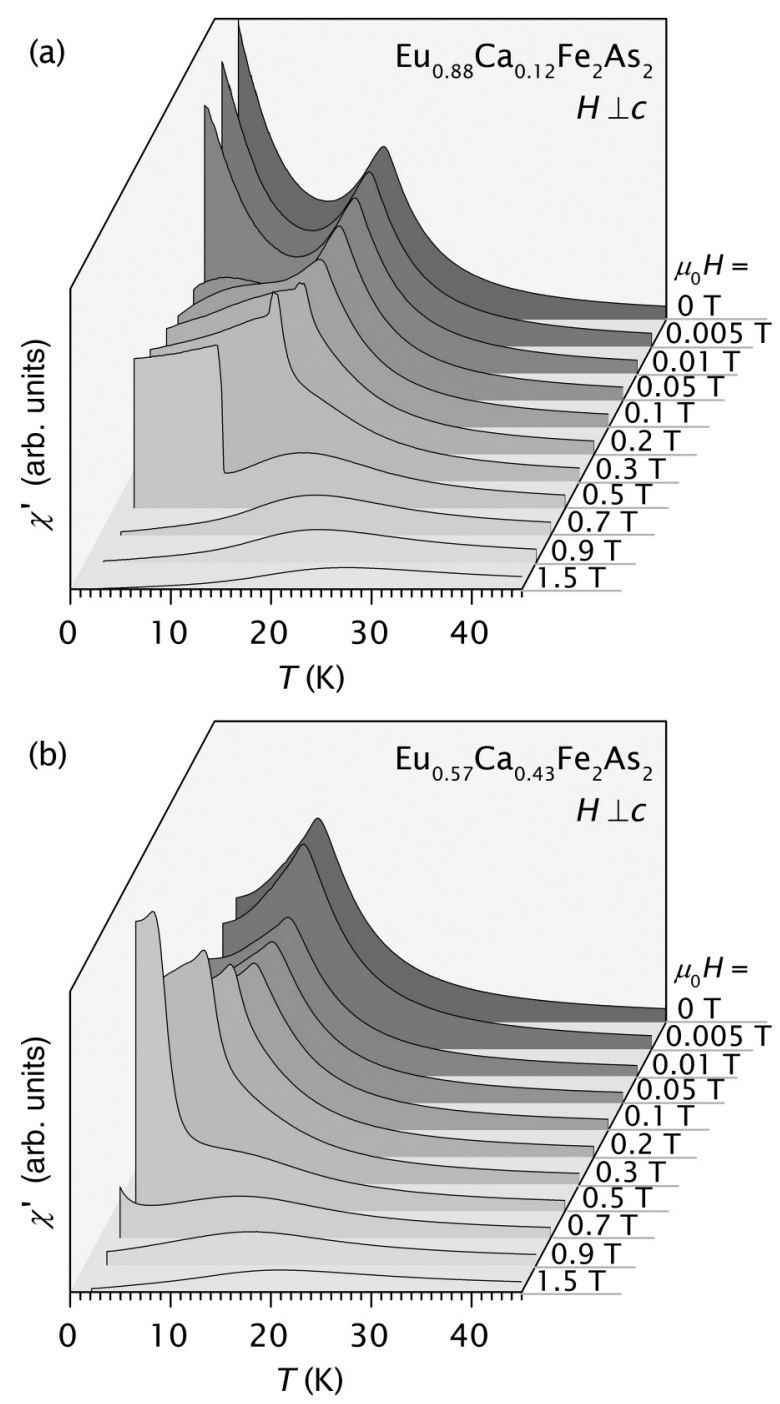

FIG. 7. Temperature dependencies of the real part of ac susceptibility $\chi^{\prime}$ of (a) $\mathrm{Eu}_{0.88} \mathrm{Ca}_{0.12} \mathrm{Fe}_{2} \mathrm{As}_{2}$ and (b) $\mathrm{Eu}_{0.57} \mathrm{Ca}_{0.43} \mathrm{Fe}_{2} \mathrm{As}_{2}$ measured in several external magnetic fields $\left(\mu_{0} H\right)$ applied perpendicular to the crystallographic $c$ axis.

At temperatures below $T_{\mathrm{N}}$ and for fields below $0.1 \mathrm{~T}$ both the real and imaginary part of the $\mathrm{Eu}_{0.88} \mathrm{Ca}_{0.12} \mathrm{Fe}_{2} \mathrm{As}_{2}$ susceptibility increase (see Fig. 8). Such behavior in a magnetically ordered state suggests the existence of additional phase transitions, especially since in the case of the P-doped $\mathrm{EuFe}_{2} \mathrm{As}_{2}-$ based compounds a spin-glass transition was observed in an already magnetically ordered state [35].

The zero-field $\left(\mu_{0} H=0 \mathrm{~T}\right)$ ac-susceptibility data collected at different frequencies reveal that although the imaginary part increases slightly at higher frequencies, the real part invariably shows the same temperature dependence (cf. Fig. 8). Additionally, constructed $\chi^{\prime \prime}\left(\chi^{\prime}\right)$ plots (see the Supplemental Material [36]) for investigated compounds do not show a semicircular shape expected for a spin-glass system [37,38], hence ruling out a spin-glass transition at low temperatures.

Taking into account the magnetization measurement results (see Sec. IIIC), we propose that the observed increase of ac susceptibility at low temperatures is possibly associated with the detwinning processes. Such suggestion is further supported 


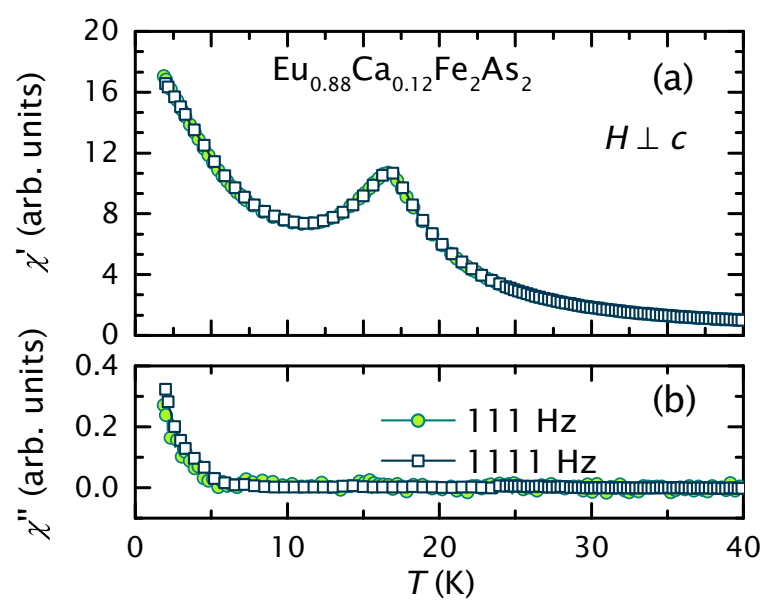

FIG. 8. Temperature dependence of (a) real and (b) imaginary parts of ac susceptibility of $\mathrm{Eu}_{0.88} \mathrm{Ca}_{0.12} \mathrm{Fe}_{2} \mathrm{As}_{2}$ measured in zero external magnetic field, using a driving field of $\mu_{0} H_{\mathrm{ac}}=10 \mathrm{mT}(\perp c)$ with frequencies of 111 and $1111 \mathrm{~Hz}$.

by the ac-susceptibility measurements of the $\mathrm{EuFe}_{2} \mathrm{As}_{2}$ parent compound (with an A-type magnetic structure), which reveal exactly the same behavior as in the $12 \%$ Ca-doped compound, i.e., a significant increase in the real and imaginary parts of ac susceptibility below the AF transition (see the Supplemental Material [36] for more details).

On the other hand, such behavior was not observed in Codoped compounds with a canted-AF structure [5,11].

Hence, we propose that the observed increase of susceptibility with decreasing temperature below $T_{\mathrm{N}}$ in both $\mathrm{EuFe}_{2} \mathrm{As}_{2}$ and $\mathrm{Eu}_{0.88} \mathrm{Ca}_{0.12} \mathrm{Fe}_{2} \mathrm{As}_{2}$ is associated with the detwinning processes, as indicated by the magnetization measurements.

\section{E. Magnetic phase diagrams}

Based on the above bulk measurements of $\mathrm{Eu}_{0.88} \mathrm{Ca}_{0.12} \mathrm{Fe}_{2} \mathrm{As}_{2}$ and $\mathrm{Eu}_{0.57} \mathrm{Ca}_{0.43} \mathrm{Fe}_{2} \mathrm{As}_{2}$, the respective magnetic-phase diagrams were constructed for fields applied both parallel and perpendicular to the $c$ axis (see Fig. 9). The vertical dashed-dotted lines represent the transition from the paramagnetic (PM) to the Fe-SDW transition. The curves corresponding to the AF to FI-FM transition, related to the $\mathrm{Eu}^{2+}$ magnetic moments, were constructed by taking the $H_{\mathrm{cr}}$ (triangles) and $T_{\mathrm{N}}$ (circles) values, calculated from the dc magnetization data, and the $T_{\mathrm{N}}$ (squares) from ac susceptibility. The transition from the paramagnetic phase of the Eu subsystem (Eu-PM) to the ordered FI-FM phase (diamonds) was determined based on the ac-susceptibility values. The dashed lines on the diagrams represent guides for the eye.

The presented phase diagrams reveal a rather anisotropic behavior. Thus, at $2 \mathrm{~K}$, in both cases the $H_{\mathrm{cr}}$ values for $H \| c$ (Fig. 9, right column) are almost twice the $H_{\mathrm{cr}}$ values obtained for $H \perp c$ (Fig. 9, left column). By comparing instead the $H_{\text {cr }}$ values of the two compounds (top vs bottom panels in Fig. 9) we deduce that the RKKY interactions become weaker upon doping, since smaller magnetic fields have to be applied to reorient the nearly 50\%-doped system from the AF to the FI-FM state with respect to the $12 \% \mathrm{Ca}$-doping case.
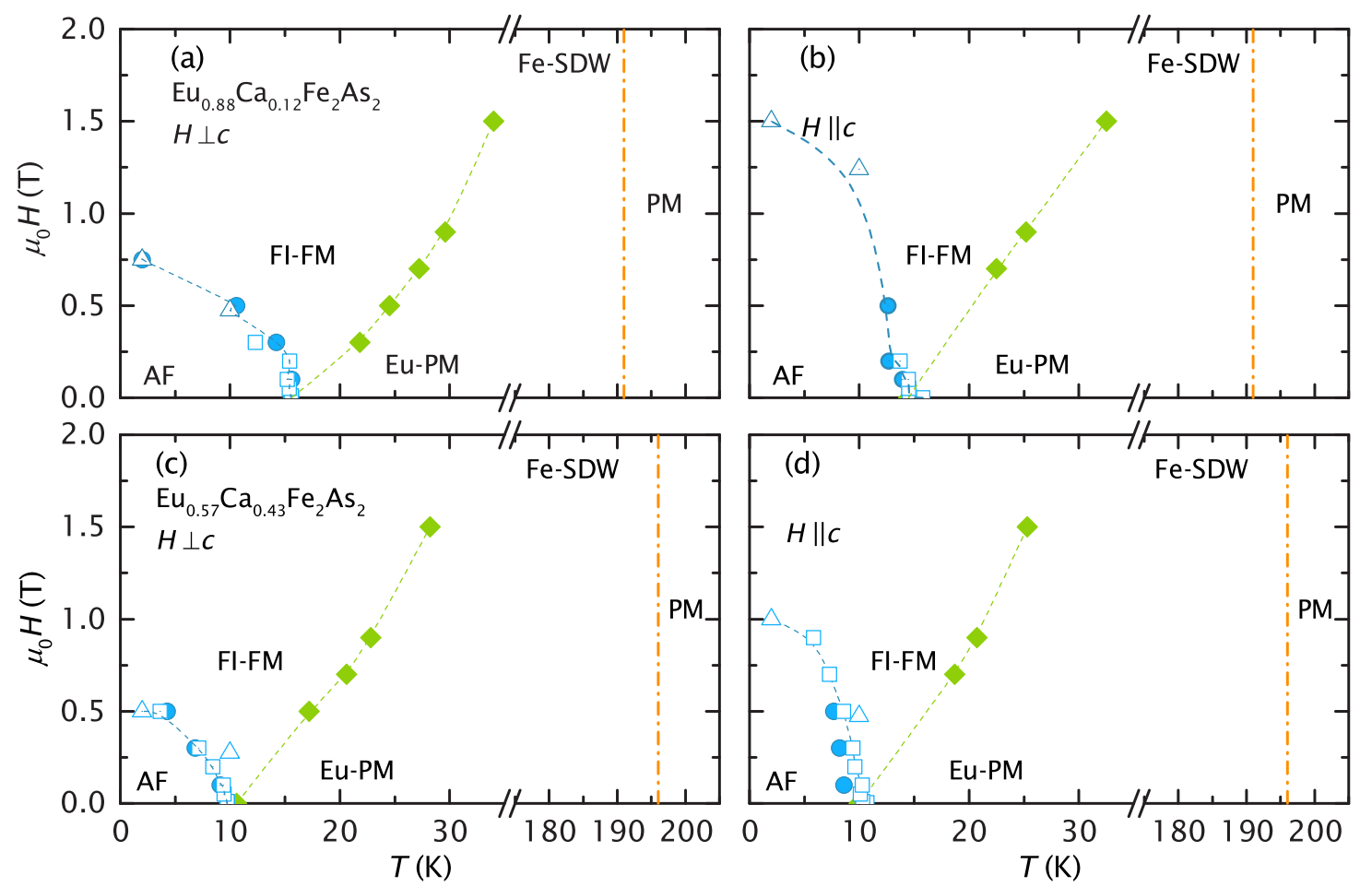

FIG. 9. Magnetic phase diagrams of [(a) and (b) - top panels] $\mathrm{Eu}_{0.88} \mathrm{Ca}_{0.12} \mathrm{Fe}_{2} \mathrm{As}_{2}$ and [(c) and (d) - bottom panels] $\mathrm{Eu}_{0.57} \mathrm{Ca}_{0.43} \mathrm{Fe}_{2} \mathrm{As}_{2}$ in magnetic fields applied (a) and (c) perpendicular and (b) and (d) parallel to the $c$ axis (see text for details). Dashed lines are guides for the eye. 


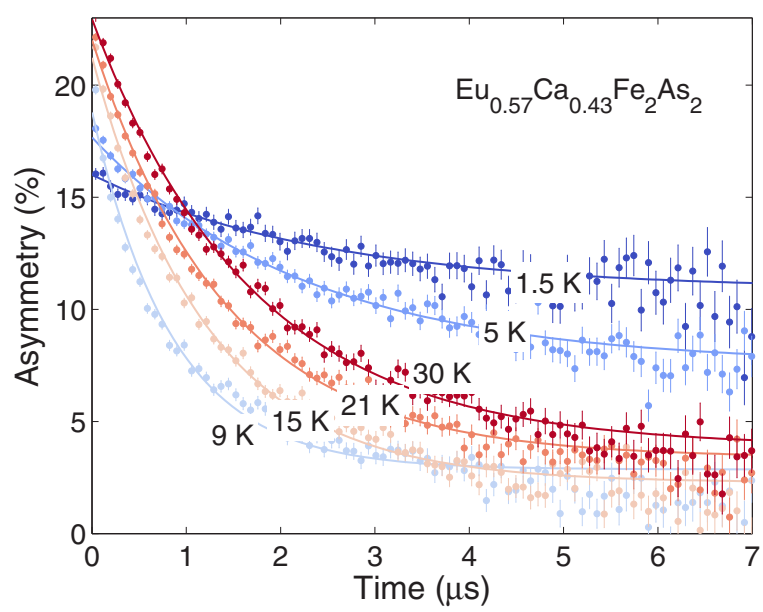

FIG. 10. Time-domain zero-field $\mu \mathrm{SR}$ asymmetry spectra of $\mathrm{Eu}_{1-x} \mathrm{Ca}_{x} \mathrm{Fe}_{2} \mathrm{As}_{2}$ for $x=0.43$ at selected temperatures observed on a long timescale. Solid curves represent fits by means of the relaxation function (2).

\section{F. Muon-spin relaxation}

Spin-polarized muons are widely used as microscopic probes of magnetism [39,40]. Once implanted in matter and thermalized at interstitial lattice sites, they act as probes of the local magnetic field, the muon-spin precession frequency being $\nu=\gamma_{\mu} /(2 \pi) B_{\text {loc }}$, with $\gamma_{\mu}=2 \pi \times 135.53 \mathrm{MHz} / \mathrm{T}$, the muon gyromagnetic ratio. Since the final signal is carried by energetic decay positrons (emitted preferentially along the muon-spin direction), $\mu \mathrm{SR}$ enables the investigation of materials even in the absence of applied fields.

To investigate the magnetic behavior of the Ca-doped $\mathrm{EuFe}_{2} \mathrm{As}_{2}$ system with temperature, muons with spins parallel to the $c$ axis were implanted into the samples with $x(\mathrm{Ca})=$ 0.12 and 0.43 , representative of a weak and strong dilution of the $\mathrm{Eu}^{2+}$ magnetic lattice, respectively. Typical time-domain asymmetry spectra for the $x=0.43$ case, collected upon warming the sample, are shown in Fig. 10. At first sight, no asymmetry oscillations are observed on a long timescale at any of the temperatures shown, either above or below $T_{\mathrm{N}}=11 \mathrm{~K}$. Except for a higher $T_{\mathrm{N}}$, similar results are obtained also in the less diluted $x=0.12$ case. In both cases, such an apparent lack of oscillations on either side of $T_{\mathrm{N}}$ is due to rather different reasons. For temperatures above $T_{\mathrm{N}}$, a smooth decay starting with a full initial asymmetry (of $22 \%$ at $30 \mathrm{~K}$ ) corresponds to the typical behavior of fluctuating paramagnetic moments $\left(\mathrm{Eu}^{2+}\right.$ in our case). Below $T_{\mathrm{N}}$ instead, a significant drop in the initial asymmetry (reaching $16 \%$ at $1.5 \mathrm{~K}$ ) reflects the onset of a long-range magnetic order. By limiting the observation time window to $0.2 \mu \mathrm{s}$, one can clearly see the oscillations in the magnetically ordered phase [see Fig. 11(a)]. The rather large $\mathrm{Eu}^{2+}$ magnetic moments $\left(\sim 7 \mu_{\mathrm{B}}\right)$ imply significant internal fields (above $0.4 \mathrm{~T}$ ) and, hence, fast precessions, which then disappear above $T_{\mathrm{N}}^{\mathrm{Eu}}$.

A comparison of the forward-backward (FB) vs up-down (UD) detector pairs (Fig. 11) is quite informative. Given the single-crystal nature of our samples, it allows us to infer also the orientation of the $\mathrm{Eu}^{2+}$ and $\mathrm{Fe}^{2+}$ magnetic moments in their respective ordered phases. Actually the $\mu \mathrm{SR}$ signal can only
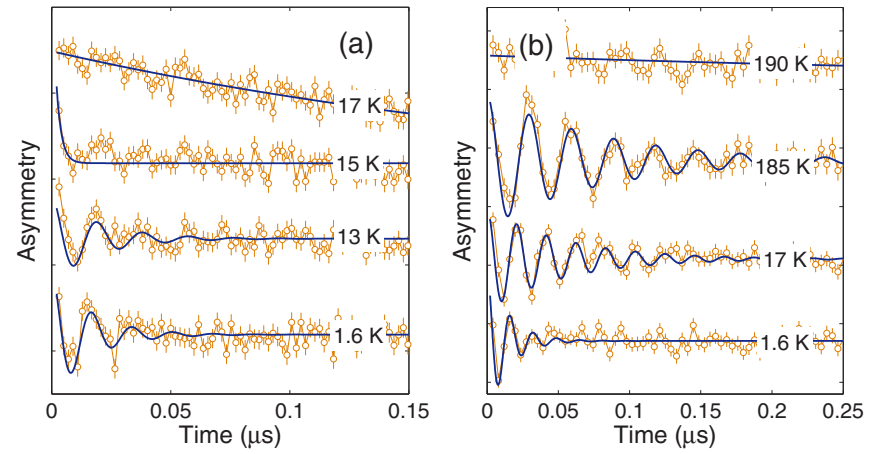

FIG. 11. Comparison of the short-time ZF- $\mu$ SR asymmetries for $x=0.12$, as measured in the FB (a) and UD (b) detectors. While in the first case the oscillations disappear above the $T_{\mathrm{N}}^{\mathrm{Eu}}$, in the second case, they persist up to the $T_{\mathrm{N}}^{\mathrm{Fe}}$. The observed spectra are compatible with the iron and europium magnetic moments lying in the $a b$ plane (see text).

give indications about the local magnetic field at the muon site, since it does not contain direct information on the magnetic moment directions. However, by taking into account the high symmetry of the muon stopping site (most likely close to the Fe planes at (1/2, 1/2, 0.22) - see, e.g., Ref. [42] and Fig. 12), one can still draw conclusions on the direction of magnetic moments for both $\mathrm{Fe}^{2+}$ and $\mathrm{Eu}^{2+}$.

The incoming muons have spins almost parallel to the $c$ axis. A lack of oscillations above $T_{\mathrm{N}}^{\mathrm{Eu}}$ in the $\mathrm{FB}$ pair, while they still persist in the UD detectors, implies that in the long-range ordered SDW phase the iron moments align in the $a b$ plane. Indeed, each muon has four nearest $\mathrm{Fe}^{2+}$ ions which, considering the $\boldsymbol{k}=(1,0,1)$ SDW propagation vector [6], implies two groups of counteraligned moments $\boldsymbol{m}$ (see

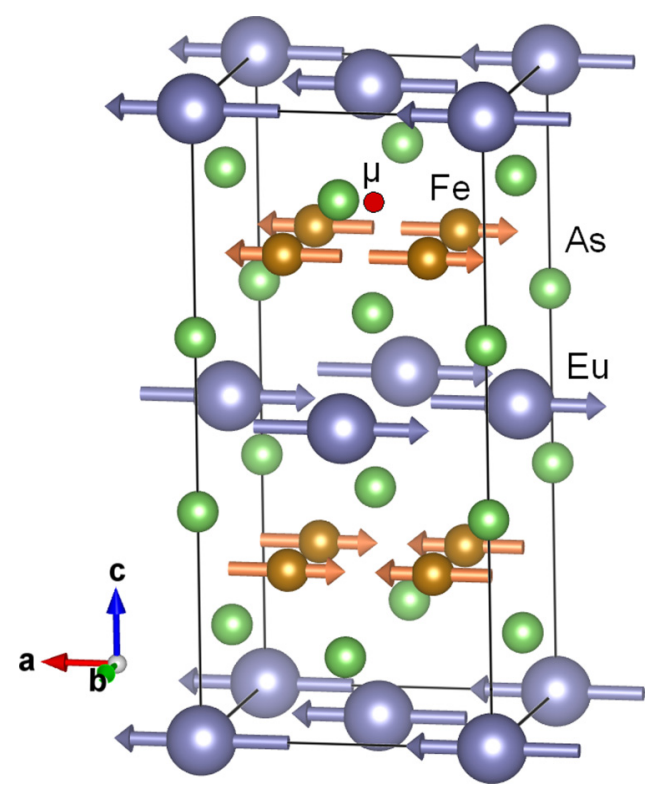

FIG. 12. The magnetic structure of Ca-doped $\mathrm{EuFe}_{2} \mathrm{As}_{2}$, as inferred from $\mu \mathrm{SR}$ data, is most likely the same as that of the $\mathrm{EuFe}_{2} \mathrm{As}_{2}$ parent compound. The red circle shows a possible (high-symmetry) muon stopping site. Drawing produced using VESTA [41]. 
Fig. 12). For symmetry reasons, the contributions to the local field at the muon site due to the $c$ component of $\boldsymbol{m}$ cancel out, whereas the in-plane component gives rise to a local dipolar field along the $c$ axis. By observing (below $T_{\mathrm{SDW}}$ ) a local field along $c$, we therefore infer that the $\mathrm{Fe}^{2+}$ moments should lie in the $a b$ plane.

As for the europium moments, since $T_{\mathrm{N}}^{\mathrm{Eu}}$ is lower than $T_{\mathrm{N}}^{\mathrm{Fe}}$, one cannot unambiguously determine their orientations, since below $T_{\mathrm{N}}^{\mathrm{Eu}}$ muons are simultaneously affected by both magnetic systems. However, compatibly with the data shown in Fig. 11(a), we expect $\mathrm{Eu}^{2+}$ moments most likely to lie in the $a b$ plane. Indeed, each muon has a single nearest $\mathrm{Eu}^{2+}$ neighbor, shifted along the $c$ direction. If the $\mathrm{Eu}^{2+}$ moment would lie along the $c$ direction, the dipolar field at the muon site would be antiparallel to the moment and along $c$ (which is not our case). If the $\mathrm{Eu}^{2+}$ moments would lie in the $a b$ plane, the dipolar field would still be antiparallel, but now in the $a b$ plane. Since below $T_{\mathrm{N}}$ we observe a local field in the $a b$ plane, we conclude that the $\mathrm{Eu}^{2+}$ magnetic moments lie also in the $a b$ plane. The above conclusions are compatible with neutron diffraction results on single crystals of $\mathrm{EuFe}_{2} \mathrm{As}_{2}$, where both $\mathrm{Eu}^{2+}$ and $\mathrm{Fe}^{2+}$ moments are shown to align along the $a$ axis (at $2.5 \mathrm{~K}$ ).

From the above discussion (and a comparison of Figs. 10 and 11), it is clear that the considered timescale determines the choice of a suitable fitting function: oscillatory for short times and slowly decaying at long times. The typical time-domain $\mu$ SR spectra reported in Fig. 10 show a strongly temperaturedependent decay which was fitted using the following combined function:

$$
A(t)=A_{0}\left[\alpha e^{-\lambda_{T} t} \cos \left(\gamma_{\mu} B_{i} t+\phi\right)+\beta e^{-\lambda_{L} t}\right] .
$$

Here $\alpha$ and $\beta=1-\alpha$ are the oscillating (i.e., transverse) and nonoscillating (i.e., longitudinal) fractions of the muon signal, respectively, whose initial total asymmetry is $A_{0}$. Accordingly, the two decay functions are denoted as $\lambda_{T}$ and $\lambda_{L}$. The initial precession angle is denoted by $\phi$, while $\gamma_{\mu}$ is the muon gyromagnetic ratio. For the ideal case of a fully magnetic polycrystalline sample one expects $\alpha=2 / 3$ and $\beta=1 / 3$ (since statistically one third of the times the muon spin does not precess, being parallel to the local magnetic field $B_{i}$ ). Similar values for the two fractions have been found also for single crystals [26], or for partially aligned mosaics (as in our case see discussion below).

As already mentioned, the apparent reduction of the longitudinal asymmetry $\beta A_{0}$ (obtained by considering the long-time $\mu$ SR spectra - see Fig. 10) is a consequence of the onset of the $\mathrm{Eu}^{2+}$ magnetic order below $T_{\mathrm{N}}$. The full recovery of asymmetry to its total value of approximately $25 \%$ occurs only in the paramagnetic phase (i.e., above $T_{c} \sim 190 \mathrm{~K}$ ). However, data show that most of the asymmetry is recovered already above $T_{\mathrm{N}}$. Considering this and the rather similar oscillating fractions $\alpha$ in a powder and in a mosaic sample, one can attempt an evaluation of the magnetic fraction $V_{M}$ as a function of doping and temperature by means of $V_{M}(T)=3 / 2\left(1-a_{L}\right) \times 100 \%$ [43]. The resulting $V_{M}(T)$ values for the pristine and Casubstituted $\mathrm{EuFe}_{2} \mathrm{As}_{2}$ samples are shown in Fig. 13.

We note that all the considered samples are fully magnetically ordered at low temperature $\left(V_{M}=100 \%\right)$. The average Néel temperature and the corresponding transition width $\Delta$

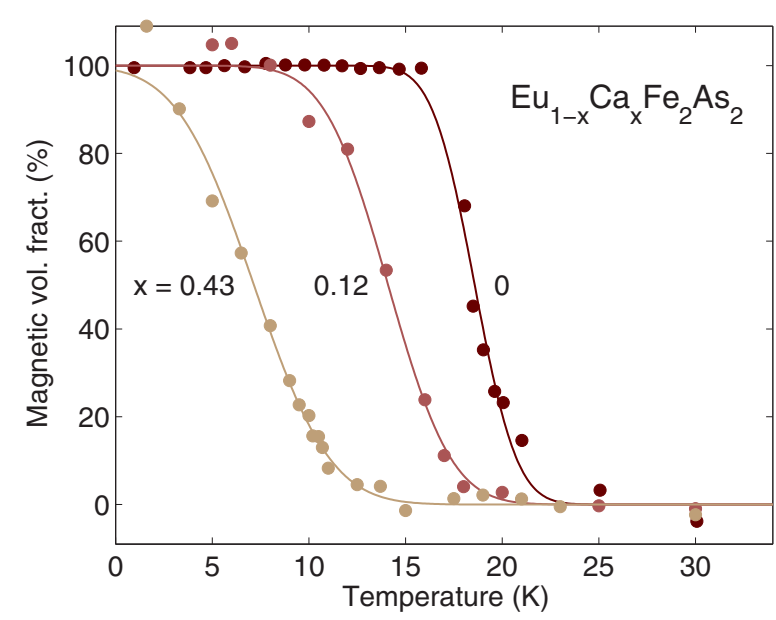

FIG. 13. Magnetic volume fraction vs temperature for samples with $x(\mathrm{Ca})=0,0.12$ and 0.43 , as extracted from the longitudinal component of $\mu \mathrm{SR}$ data and fitted by means of Eq. (3). The data for the $x=0$ case were obtained from Ref. [20].

were obtained by fitting the $V_{M}(T)$ data with the phenomenological function [43]:

$$
V_{M}(T)=\frac{1}{2}\left[1-\operatorname{erf}\left(\frac{T-T_{\mathrm{N}}}{\sqrt{2} \Delta}\right)\right] .
$$

As can be seen from Fig. 13, as the Ca content increases there is a clear decrease of $T_{\mathrm{N}}$ and a simultaneous broadening of the transition (the curves become smoother). These results are in very good agreement with our magnetometry data. In addition, they show that a high $\mathrm{Ca}$ substitution rate corresponds to an increased degree of disorder, here measured by $\Delta$. The detailed fit values are reported in Table II.

Although strictly speaking the relaxation rate is not an order parameter, by considering the fit parameter $\lambda_{L}$, we can still obtain information on the critical dynamics, as suggested by the sharp cusps observed at $T_{\mathrm{N}}$ (diamond symbols in Figs. 14 and 15). The $T$ dependence of $\lambda_{L}$, both above and below $T_{\mathrm{N}}$, has been described by means of a critical-exponent behavior $\lambda(T)=\lambda_{0}\left|1-T / T_{\mathrm{N}}\right|^{-w}[44,45]$, with the expected exponent value for a $3 \mathrm{D}$ ferromagnet $w \approx 1.05$. The fit curves, shown with solid lines in Fig. 14(b), have rather different exponents above and below $T_{\mathrm{N}}$. Thus for $T<T_{\mathrm{N}}$ we find $w \simeq 0.84(3)$, whereas for $T>T_{\mathrm{N}}$ we obtain $w \simeq 0.25(2)$. While the former value is not far from that expected at a magnetic transition, the latter is much smaller and remains so even when restricting the fit range. Such anomalously small $w$ values above $T_{\mathrm{N}}$ have been observed also elsewhere (see, e.g., Ref. [20]), where $w$ was found to be 0.28 . It has been suggested that muon-lattice

TABLE II. Magnetic properties of $\mathrm{Eu}_{1-x} \mathrm{Ca}_{x} \mathrm{Fe}_{2} \mathrm{As}_{2}$ samples as a function of Ca substitution.

\begin{tabular}{lrcc}
\hline \hline$x(\mathrm{Ca})$ & $T_{\mathrm{N}}(\mathrm{K})$ & $\Delta(\mathrm{K})$ & Magn. frac. (\%) \\
\hline 0 & $18.6(1)$ & $1.7(3)$ & $99(4)$ \\
$0.12(1)$ & $15.1(3)$ & $2.6(3)$ & $100(5)$ \\
$0.43(1)$ & $7.2(4)$ & $3.1(4)$ & $100(8)$ \\
\hline \hline
\end{tabular}




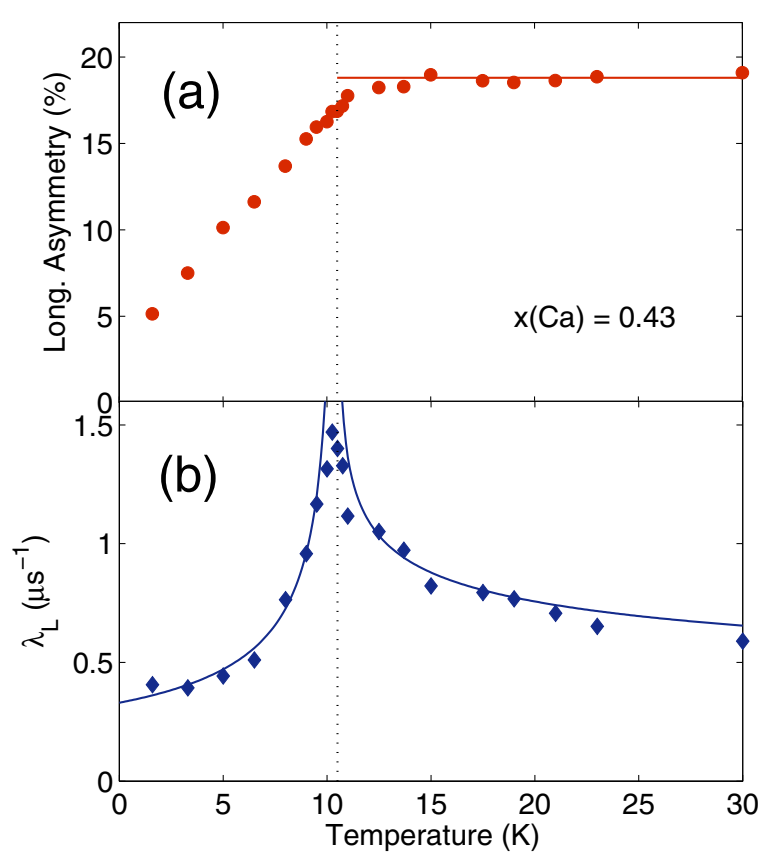

FIG. 14. Fit parameters of the long-time $\mu$ SR spectra vs temperature for the $x=0.43$ case: (a) Appararent longitudinal muon-spin asymmetry at long timescales (c.f. Fig. 10) at zero field and (b) longitudinal decay coefficient. Both parameters show marked features (slope change or cusp) in concomitance with the $\mathrm{Eu}^{2+} \mathrm{AF}$ phase transition at $T_{\mathrm{N}}=11 \mathrm{~K}$.

dipolar interactions, known to strongly affect the paramagnetic critical dynamics near a magnetic transition point, could be responsible for the observed low value of the $w$ exponent $[44,45]$.

By considering now the short-time $\mu$ SR data (see Fig. 11) we can follow the temperature evolution of the internal magnetic field, as sensed at the muon stopping sites. The resulting fit parameters in the $x=0.12$ case, obtained by means of Eq. (2), are shown in Figs. 15(a) and 15(b) for the UD and FB detector pairs, respectively. In comparison with the longitudinal relaxation data [see Fig. 14(b)] the transverse relaxation is more than two orders(!) of magnitude larger. Similarly large relaxation rates have been observed also in the isoelectronically doped $\mathrm{EuFe}_{2}\left(\mathrm{As}_{1-x} \mathrm{P}_{x}\right)_{2}$ compound [26].

As discussed above, the UD detector dataset allows us to follow both the Eu- and Fe-related magnetic transitions, distinctly seen at $T_{\mathrm{N}}=15 \mathrm{~K}$ and $T_{\mathrm{SDW}}=190 \mathrm{~K}$, respectively, in the field profiles and in the muon relaxation rates [see Fig. 15(a)]. By converse, the FB detector dataset is limited to the $\mathrm{Eu}^{2+}$ transition only. Analogously to the longitudinal relaxation, also the transverse relaxation data show a critical-exponent behavior $\lambda(T)=\lambda_{0}\left|1-T / T_{\mathrm{N}}\right|^{-w}$, once more with rather skewed exponent values above $T_{\mathrm{N}}$.

As for the internal field dependence with $T$, the very sharp increase of $B_{i}$ at $T_{\mathrm{SDW}}$ is suggestive of a first-order phase transition. As the temperature is lowered, the internal field tends to saturate at approximately $0.35 \mathrm{~T}$. At $T_{\mathrm{N}}$ another field jump appears, indicative of the magnetic ordering of $\mathrm{Eu}^{2+}$ moments. Each of these transitions can be modeled by the
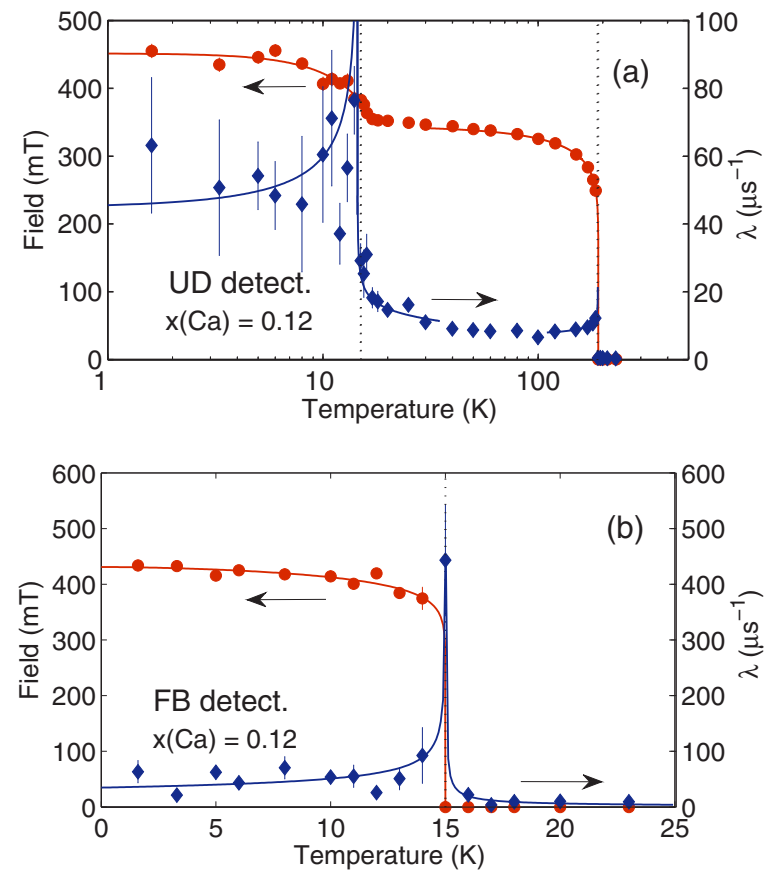

FIG. 15. (a) Short-time ZF- $\mu$ SR fit parameters vs temperature for the $x=0.12$ case, showing the Eu- and Fe-related phase transitions at 15 and $190 \mathrm{~K}$, respectively (dashed lines). Since data refer to the UD detector pair, both transitions are observed. The internal magnetic field (circles) was determined from the $\mu \mathrm{SR}$ oscillation frequency. The decay rate (diamonds) shows marked peaks in concomitance with the two magnetic phase transitions. (b) Same as in (a), but data refer to the FB detector pair. The observed field (left scale) goes abruptly to zero at $T_{\mathrm{N}}^{\mathrm{Eu}}$, where the decay rate (right scale) exhibits a very marked divergence.

phenomenological model $[46,47]$ :

$$
B_{i}(T)=\sum_{n=1}^{2} B_{i}^{n}(0)\left[1-\left(\frac{T}{T_{c}^{n}}\right)^{\gamma_{n}}\right]^{\delta_{n}},
$$

where $B_{i}^{n}(0)$ are the internal magnetic fields at zero temperature and $\gamma_{n}$ and $\delta_{n}$ are two empirical parameters, whose typical fit values are $\gamma_{n} \sim 1.5$ and $\delta_{n} \sim 0.25$ (with error bars of approximately 0.8 ).

For both samples, none of the internal magnetic fields seems to track an ideal mean-field type curve, which represents the exact solution in case of an infinite-range (anti)ferromagnetic order and corresponds to $\delta_{n} \sim 0.5$. The reason for such discrepancy might reflect the occurrence of a magnetic (AF) order within an already ordered (SDW) phase.

\section{SUMMARY AND CONCLUSIONS}

Our study suggests that similarly to the $\mathrm{EuFe}_{2} \mathrm{As}_{2}$ parent compound, $\mathrm{Eu}_{0.88} \mathrm{Ca}_{0.12} \mathrm{Fe}_{2} \mathrm{As}_{2}$ and (most likely) $\mathrm{Eu}_{0.57} \mathrm{Ca}_{0.43} \mathrm{Fe}_{2} \mathrm{As}_{2}$ undergo analogous detwinning processes. Some differences observed between the two Ca-doped compounds are possibly due to the different coupling strengths between the Fe and Eu subsystems, associated with the different Eu concentration in the two cases. 
The $\mu \mathrm{SR}$ results offer a local-probe perspective on the Ca-doped $\mathrm{EuFe}_{2} \mathrm{As}_{2}$ system, e.g., implanted muons indicate that in the magnetically ordered phase both the $\mathrm{Fe}^{2+}$ and $\mathrm{Eu}^{2+}$ magnetic moments align in the $a b$ plane. The latter is also supported by magnetometry results, which suggest an A-type $\mathrm{AF}$ structure for the $\mathrm{Eu}^{2+}$ magnetic moments in the Cadoped compounds, the same as in the $\mathrm{EuFe}_{2} \mathrm{As}_{2}$ case. At low temperatures, both samples show $100 \%$ magnetic fractions, but a dilution of the $\mathrm{Eu}^{2+}$ sublattice implies a decrease of $T_{\mathrm{N}}$ and a broadening of the transition. In both cases, muons detect magnetic fields of approximately $450 \mathrm{mT}$ in the ordered phase, with the muon field profiles and relaxation rates showing distinct features at the respective phase transitions of the two magnetic sublattices. The presence of an AF order, even in the nearly $50 \%$ diluted system, excludes direct interactions among $\mathrm{Eu}^{2+}$ moments as responsible for the observed 3D magnetic order and suggests instead the indirect RKKY couplings to be the key interactions. Such a conclusion is supported also by theoretical calculations, as shown, e.g., in Ref. [48]. The significant reduction of $T_{\mathrm{N}}$ and $H_{\mathrm{cr}}$ in $\mathrm{EuFe}_{2} \mathrm{As}_{2}$ upon Euby-Ca substitution indicates a clear weakening of the RKKYmediated magnetic interactions among $\mathrm{Eu}^{2+}$ moments. This result could be rationalized by considering the direct influence of local on-site substitutions and is in clear contrast with the outcome of substitutions in the FeAs planes (such as, e.g.,
Co-for-Fe), for which the temperature of the AF ordering of $\mathrm{Eu}^{2+}$ moments remains mostly unchanged upon doping [32].

Finally, we observe also a small change of $T_{\mathrm{SDW}}$ values upon doping, which might suggest some degree of interaction between the Eu and Fe layers. This is consistent with theoretical predictions, which indicate that the Eu order might be influenced by the Fe magnetism $[31,48]$. However, one cannot exclude that a $T_{\mathrm{SDW}}$ change might arise simply because of a change in the lattice parameters or other processes. Therefore, the investigation of similar compounds but with smaller or (preferably) with no changes of lattice parameters, will be helpful to solve this issue.

Given the interesting zero-pressure results reported above, future high-pressure $\mu$ SR investigations of Ca-doped $\mathrm{EuFe}_{2} \mathrm{Ca}_{2}$ are promising, since the Ca-doped $\mathrm{EuFe}_{2} \mathrm{As}_{2}$ system is expected to become a superconductor above 1.5-2 GPa.

\section{ACKNOWLEDGMENTS}

We thank H. Luetkens and J. Barker for the experimental support at PSI. This work is partially based on experiments performed at the Swiss Muon Source $\mathrm{S} \mu \mathrm{S}$, Paul Scherrer Institute, Villigen, Switzerland and was financially supported in part by the Schweizerische Nationalfonds zur Förderung der Wissenschaftlichen Forschung (SNF), Grant No. 200021-169455.
[1] M. B. Maple, Interplay between superconductivity and magnetism, Phys. B 215, 110 (1995).

[2] A. B. Vorontsov, M. G. Vavilov, and A. V. Chubukov, Interplay between magnetism and superconductivity in the iron pnictides, Phys. Rev. B 79, 060508 (2009).

[3] S. Sanna, P. Carretta, P. Bonfà, G. Prando, G. Allodi, R. De Renzi, T. Shiroka, G. Lamura, A. Martinelli, and M. Putti, Correlated Trends of Coexisting Magnetism and Superconductivity in Optimally Electron-Doped Oxypnictides, Phys. Rev. Lett. 107, 227003 (2011).

[4] S. Zapf and M. Dressel, Europium-based iron pnictides: A unique laboratory for magnetism, superconductivity and structural effects, Rep. Prog. Phys. 80, 016501 (2017).

[5] V. H. Tran, Z. Bukowski, L. M. Tran, and A. J. Zaleski, The electronic phase diagrams of the $\mathrm{Eu}\left(\mathrm{Fe}_{0.81} \mathrm{Co}_{0.19}\right)_{2} \mathrm{As}_{2}$ superconductor, New J. Phys. 14, 073052 (2012).

[6] Y. Xiao, Y. Su, M. Meven, R. Mittal, C. M. N. Kumar, T. Chatterji, S. Price, J. Persson, N. Kumar, S. K. Dhar, A. Thamizhavel, and Th. Brueckel, Magnetic structure of $\mathrm{EuFe}_{2} \mathrm{As}_{2}$ determined by single-crystal neutron diffraction, Phys. Rev. B 80, 174424 (2009).

[7] T. Terashima, M. Kimata, H. Satsukawa, A. Harada, K. Hazama, S. Uji, H. S. Suzuki, T. Matsumoto, and K. Murata, $\mathrm{EuFe}_{2} \mathrm{As}_{2}$ under high pressure: An antiferromagnetic bulk superconductor, J. Phys. Soc. Jpn. 78, 083701 (2009).

[8] C. F. Miclea, M. Nicklas, H. S. Jeevan, D. Kasinathan, Z. Hossain, H. Rosner, P. Gegenwart, C. Geibel, and F. Steglich, Evidence for a reentrant superconducting state in $\mathrm{EuFe}_{2} \mathrm{As}_{2}$ under pressure, Phys. Rev. B 79, 212509 (2009).

[9] N. V. Morozova, A. E. Karkin, S. V. Ovsyannikov, Y. A. Umerova, V. V. Shchennikov, R. Mittal, and A. Thamizhavel, Electronic transport properties of $\mathrm{MFe}_{2} \mathrm{As}_{2}(\mathrm{M}=\mathrm{Ca}, \mathrm{Eu}, \mathrm{Sr})$ at ambient and high pressures up to $20 \mathrm{GPa}$, Supercond. Sci. Technol. 28, 125010 (2015).

[10] S. Jiang, H. Xing, G. Xuan, Z. Ren, C. Wang, Z. A. Xu, and G. Cao, Superconductivity and local-moment magnetism in $\mathrm{Eu}\left(\mathrm{Fe}_{0.89} \mathrm{Co}_{0.11}\right)_{2} \mathrm{As}_{2}$, Phys. Rev. B 80, 184514 (2009).

[11] V. H. Tran, T. A. Zaleski, Z. Bukowski, L. M. Tran, and A. J. Zaleski, Tuning superconductivity in $\mathrm{Eu}\left(\mathrm{Fe}_{0.81} \mathrm{Co}_{0.19}\right)_{2} \mathrm{As}_{2}$ with magnetic fields, Phys. Rev. B 85, 052502 (2012).

[12] Z. Ren, Q. Tao, S. Jiang, C. Feng, C. Wang, J. Dai, G. Cao, and Z. $\mathrm{Xu}$, Superconductivity Induced by Phosphorus Doping and Its Coexistence with Ferromagnetism in $\mathrm{EuFe}_{2}\left(\mathrm{As}_{0.7} \mathrm{P}_{0.3}\right)_{2}$, Phys. Rev. Lett. 102, 137002 (2009).

[13] W.-H. Jiao, H.-F. Zhai, J.-K. Bao, Y.-K. Luo, Q. Tao, C.-M. Feng, Z.-A. Xu, and G. Cao, Anomalous critical fields and the absence of Meissner state in $\mathrm{Eu}\left(\mathrm{Fe}_{0.88} \mathrm{Ir}_{0.12}\right)_{2} \mathrm{As}_{2}$ crystals, New J. Phys. 15, 113002 (2013).

[14] U. B. Paramanik, D. Das, R. Prasad, and Z. Hossain, Reentrant superconductivity in $\mathrm{Eu}\left(\mathrm{Fe}_{1-x} \mathrm{Ir}_{x}\right)_{2} \mathrm{As}_{2}$, J. Phys. 25, 265701 (2013).

[15] H. S. Jeevan, Z. Hossain, Deepa Kasinathan, H. Rosner, C. Geibel, and P. Gegenwart, High-temperature superconductivity in $\mathrm{Eu}_{0.5} \mathrm{~K}_{0.5} \mathrm{Fe}_{2} \mathrm{As}_{2}$, Phys. Rev. B 78, 092406 (2008).

[16] Y. Qi, Z. Gao, L. Wang, D. Wang, X. Zhang, and Y. Ma, Superconductivity at $34.7 \mathrm{~K}$ in the iron arsenide $\mathrm{Eu}_{0.7} \mathrm{Na}_{0.3} \mathrm{Fe}_{2} \mathrm{As}_{2}$, New J. Phys. 10, 123003 (2008).

[17] M. Zhang, J. J. Ying, Y. J. Yan, A. F. Wang, X. F. Wang, Z. J. Xiang, G. J. Ye, P. Cheng, X. G. Luo, J. Hu, and X. H. Chen, Phase diagram as a function of doping level and pressure in the $\mathrm{Eu}_{1-x} \mathrm{La}_{x} \mathrm{Fe}_{2} \mathrm{As}_{2}$ system, Phys. Rev. B 85, 092503 (2012).

[18] A. Błachowski, K. Ruebenbauer, J. Żukrowski, Z. Bukowski, K. Rogacki, P. J. W. Moll, and J. Karpinski, Interplay between magnetism and superconductivity in $\mathrm{EuFe}_{2-x} \mathrm{Co}_{x} \mathrm{As}_{2}$ studied by 
${ }^{57} \mathrm{Fe}$ and ${ }^{151}$ Eu Mössbauer spectroscopy, Phys. Rev. B 84, 174503 (2011).

[19] W. T. Jin, Y. Xiao, Z. Bukowski, Y. Su, S. Nandi, A. P. Sazonov, M. Meven, O. Zaharko, S. Demirdis, K. Nemkovski, K. Schmalzl, L. M. Tran, Z. Guguchia, E. Feng, Z. Fu, and Th. Brückel, Phase diagram of Eu magnetic ordering in Snflux-grown $\mathrm{Eu}\left(\mathrm{Fe}_{1-x} \mathrm{Co}_{x}\right)_{2} \mathrm{As}_{2}$ single crystals, Phys. Rev. B 94, 184513 (2016).

[20] V. K. Anand, D. T. Adroja, A. Bhattacharyya, U. B. Paramanik, P. Manuel, A. D. Hillier, D. Khalyavin, and Z. Hossain, $\mu$ SR and neutron diffraction investigations on the reentrant ferromagnetic superconductor $\mathrm{Eu}\left(\mathrm{Fe}_{0.86} \mathrm{Ir}_{0.14}\right)_{2} \mathrm{As}_{2}$, Phys. Rev. B 91, 094427 (2015).

[21] I. Nowik, I. Felner, Z. Ren, G. H. Cao, and Z. A. Xu, Coexistence of ferromagnetism and superconductivity: Magnetization and Mössbauer studies of $\mathrm{EuFe}_{2}\left(\mathrm{As}_{1-x} \mathrm{P}_{x}\right)_{2}$, J. Phys. 23, 065701 (2011).

[22] K. Komędera, A. Błachowski, K. Ruebenbauer, J. Żukrowski, S. M. Dubiel, L. M. Tran, M. Babij, and Z. Bukowski, Mössbauer study of $\mathrm{Eu}_{0.57} \mathrm{Ca}_{0.43} \mathrm{Fe}_{2} \mathrm{As}_{2}$ and $\mathrm{Eu}_{0.73} \mathrm{Ca}_{0.27}\left(\mathrm{Fe}_{0.87} \mathrm{Co}_{0.13}\right)_{2} \mathrm{As}_{2}$ : A comparison to 122 iron-based superconductors parent compounds $\mathrm{EuFe}_{2} \mathrm{As}_{2}$ and $\mathrm{CaFe}_{2} \mathrm{As}_{2}$, J. Magn. Magn. Mater. 457, 1 (2018)

[23] A. Mitsuda, T. Matoba, S. Seike, F. Ishikawa, Y. Yamada, and $\mathrm{H}$. Wada, Effect of substitution of $\mathrm{Ca}^{2+}$ for $\mathrm{Eu}^{2+}$ on pressureinduced superconductivity in $\mathrm{EuFe}_{2} \mathrm{As}_{2}$, J. Phys. Soc. Jpn. 80, SA117 (2011).

[24] A. Mitsuda, S. Seike, T. Matoba, H. Wada, F. Ishikawa, and Y. Yamada, Competition between Fe-based superconductivity and antiferromagnetism of $\mathrm{Eu}^{2+}$ in $\mathrm{Eu}_{1-x} \mathrm{Ca}_{x} \mathrm{Fe}_{2} \mathrm{As}_{2}$, J. Phys. 273, 012100 (2011).

[25] Anupam, P. L. Paulose, S. Ramakrishnan, and Z. Hossain, Doping dependent evolution of magnetism and superconductivity in $\mathrm{Eu}_{1-\mathrm{x}} \mathrm{K}_{\mathrm{x}} \mathrm{Fe}_{2} \mathrm{As}_{2}(x=0-1)$ and temperature dependence of the lower critical field $H_{\mathrm{cl}}$, J. Phys. 23, 455702 (2011).

[26] Z. Guguchia, A. Shengelaya, A. Maisuradze, L. Howald, Z. Bukowski, M. Chikovani, H. Luetkens, S. Katrych, J. Karpinski, and H. Keller, Muon-spin rotation and magnetization studies of chemical and hydrostatic pressure effects in $\mathrm{EuFe}_{2}\left(\mathrm{As}_{1-x} \mathrm{P}_{x}\right)_{2}$, J. Supercond. Nov. Magn. 26, 285 (2013).

[27] T. Goltz, S. Kamusella, H. S. Jeevan, P. Gegenwart, H. Luetkens, P. Materne, J. Spehling, R. Sarkar, and H.-H. Klauss, Interplay of magnetism and superconductivity in $\mathrm{EuFe}_{2}\left(\mathrm{As}_{1-x} \mathrm{P}_{x}\right)_{2}$ single crystals probed by muon spin rotation and ${ }^{57} \mathrm{Fe}$ Mössbauer spectroscopy, J. Phys.: Conf. Ser. 551, 012025 (2014).

[28] A. Mitsuda, T. Matoba, F. Ishikawa, Y. Yamada, and H. Wada, Pressure-induced superconductivity in $\mathrm{Eu}_{0.5} \mathrm{Ca}_{0.5} \mathrm{Fe}_{2} \mathrm{As}_{2}$ : Wide zero-resistivity region due to suppression of Eu magnetic order and chemical pressure, J. Phys. Soc. Jpn. 79, 073704 (2010).

[29] S. Jiang, Y. Luo, Z. Ren, Z. Zhu, C. Wang, X. Xu, Q. Tao, G. Cao, and Z. Xu, Metamagnetic transition in $\mathrm{EuFe}_{2} \mathrm{As}_{2}$ single crystals, New J. Phys. 11, 025007 (2009).

[30] S. Zapf, C. Stingl, K. W. Post, J. Maiwald, N. Bach, I. Pietsch, D. Neubauer, A. Löhle, C. Clauss, S. Jiang, H. S. Jeevan, D. N. Basov, P. Gegenwart, and M. Dressel, Persistent Detwinning of Iron-Pnictide $\mathrm{EuFe}_{2} \mathrm{As}_{2}$ Crystals by Small External Magnetic Fields, Phys. Rev. Lett. 113, 227001 (2014).

[31] J. Maiwald, I. I. Mazin, and Ph. Gegenwart, Microscopic Theory of Magnetic Detwinning in Iron-Based Superconductors with Large-Spin Rare Earths, Phys. Rev. X 8, 011011 (2018).
[32] Z. Guguchia, S. Bosma, S. Weyeneth, A. Shengelaya, R. Puzniak, Z. Bukowski, J. Karpinski, and H. Keller, Anisotropic magnetic order of the Eu sublattice in single crystals of $\mathrm{EuFe}_{2-x} \mathrm{Co}_{x} \mathrm{As}_{2}(x=0,0.2)$ studied by means of magnetization and magnetic torque, Phys. Rev. B 84, 144506 (2011).

[33] M. E. Fisher, Relation between the specific heat and susceptibility of an antiferromagnet, Philos. Mag. 7, 1731 (1962).

[34] J. Ballinger, L. E. Wenger, Y. K. Vohra, and A. S. Sefat, Magnetic properties of single crystal $\mathrm{EuCo}_{2} \mathrm{As}_{2}$, J. Appl. Phys. 111, 07E106 (2012).

[35] S. Zapf, H. S. Jeevan, T. Ivek, F. Pfister, F. Klingert, S. Jiang, D. Wu, P. Gegenwart, R. K. Kremer, and M. Dressel, $\mathrm{EuFe}_{2}\left(\mathrm{As}_{1-\mathrm{x}} \mathrm{P}_{\mathrm{x}}\right) 2$ : Reentrant Spin Glass and Superconductivity, Phys. Rev. Lett. 110, 237002 (2013).

[36] See Supplemental Material at http://link.aps.org/supplemental/ 10.1103/PhysRevB.98.104412 for more detailed ac susceptibility measurement results containing temperature, field, and frequency dependence .

[37] J. A. Mydosh, Spin Glasses: An Experimental Introduction (Taylor \& Francis, London, 1993).

[38] O. Petracic, S. Sahoo, Ch. Binek, W. Kleemann, J. B. Sousa, S. Cardoso, and P. P. Freitas, Cole-Cole analysis of the superspin glass system $\mathrm{Co}_{80} \mathrm{Fe}_{20} / \mathrm{Al}_{2} \mathrm{O}_{3}$, Phase Trans. 76, 367 (2003).

[39] S. J. Blundell, Spin-polarized muons in condensed matter physics, Contemp. Phys. 40, 175 (1999).

[40] A. Yaouanc and P. Dalmas de Réotier, Muon Spin Rotation, Relaxation, and Resonance: Applications to Condensed Matter (Oxford University Press, Oxford, 2011).

[41] K. Momma and F. Izumi, VESTA3 for three-dimensional visualization of crystal, volumetric and morphology data, J. Appl. Crystallogr. 44, 1272 (2011).

[42] H. Maeter, H. Luetkens, Yu. G. Pashkevich, A. Kwadrin, R. Khasanov, A. Amato, A. A. Gusev, K. V. Lamonova, D. A. Chervinskii, R. Klingeler, C. Hess, G. Behr, B. Büchner, and H.-H. Klauss, Interplay of rare earth and iron magnetism in $\mathrm{RFeAsO}(\mathrm{R}=\mathrm{La}, \mathrm{Ce}, \mathrm{Pr}$, and $\mathrm{Sm})$ : Muon-spin relaxation study and symmetry analysis, Phys. Rev. B 80, 094524 (2009).

[43] T. Shiroka, G. Lamura, S. Sanna, G. Prando, R. De Renzi, M. Tropeano, M. R. Cimberle, A. Martinelli, C. Bernini, A. Palenzona, R. Fittipaldi, A. Vecchione, P. Carretta, A. S. Siri, C. Ferdeghini, and M. Putti, Long- to short-range magnetic order in fluorine-doped CeFeAsO, Phys. Rev. B 84, 195123 (2011).

[44] A. Yaouanc, P. Dalmas de Réotier, and E. Frey, Zero-field muonspin-relaxation depolarization rate of paramagnets near the Curie temperature, Phys. Rev. B 47, 796 (1993).

[45] S. Henneberger, E. Frey, P. G. Maier, F. Schwabl, and G. M. Kalvius, Critical dynamics of a uniaxial and dipolar ferromagnet, Phys. Rev. B 60, 9630 (1999).

[46] L. P. Le, A. Keren, G. M. Luke, W. D. Wu, Y. J. Uemura, M. Tamura, M. Ishikawa, and M. Kinoshita, Searching for spontaneous magnetic order in an organic ferromagnet. $\mu \mathrm{SR}$ studies of $\beta$-phase p-NPNN, Chem. Phys. Lett. 206, 405 (1993).

[47] S. J. Blundell, P. A. Pattenden, F. L. Pratt, R. M. Valladares, T. Sugano, and W. Hayes, $\mu^{+}$SR of the organic ferromagnet p-NPNN: Diamagnetic and paramagnetic states, Europhys. Lett. 31, 573 (1995).

[48] A. Akbari, I. Eremin, and P. Thalmeier, RKKY interaction in the spin-density-wave phase of iron-based superconductors, Phys. Rev. B 84, 134513 (2011). 